\title{
Copyright
}

by

Avinash Voruganti

2009 


\section{Modeling Carrier Collaboration in Freight Networks}

by

Avinash Voruganti, B.TECH

\section{THESIS}

Presented to the Faculty of the Graduate School of The University of Texas at Austin

in Partial Fulfillment

of the Requirements

for the Degree of

\section{MASTER OF SCIENCE IN ENGINEERING}

\section{THE UNIVERSITY OF TEXAS AT AUSTIN}

August 2009 
The Thesis committee for Avinash Voruganti

Certifies that this is the approved version of the following thesis:

\section{Modeling Carrier Collaboration in Freight Networks}

APPROVED BY

SUPERVISING COMMITTEE:

Supervisor:

(S. Travis Waller)

(Randy. B. Machemehl) 
To my family, friends and teachers 


\section{Acknowledgements}

Firstly, I would like to thank my advisor Dr. Travis Waller for providing me valuable time over the past couple of years to pursue the topic of my interest. I would also like to thank Dr. Randy Machemehl for his help in reviewing my thesis.

My special thanks to Avinash Unnnikrishnan, who has played a major role in helping me develop this thesis right from formulating the problem till the finish. I am also thankful to all my fellow graduate students who have been an integral part of my life over the past two years. I would also like to thank all my friends for providing me with wonderful moments to cherish throughout my life.

Finally, I would like to thank my parents and sisters, who have always stood by my side and shared my success and failures. 


\title{
Modeling Carrier Collaboration in Freight Networks
}

\author{
Avinash Voruganti, M.S.E \\ The University of Texas at Austin, 2009
}

Supervisor: S. Travis Waller

This work presents two mechanisms for modeling alliance formation between leader carriers in a freight network for more efficient utilization of their resources: partial collaboration and complete collaboration. The performance of these alliance formation mechanisms is compared against the no collaboration case for various network topologies and demand levels. In the partial collaboration case, each leader carrier first maximizes his individual profits and leases out the residual capacity to other carriers. In the complete collaboration case all leader carriers join together to maximize the profit of the alliance. The profits are then distributed among the alliance members using the Shapley value principle. Numerical tests reveal that the topology of the network and the demand levels play an important role in determining the profits from each collaboration mechanism. It was also inferred that each of these factors also play a major role in determining the best collaboration strategy. 


\section{Table of Contents}

Acknowledgements............................................................................................................................................. v

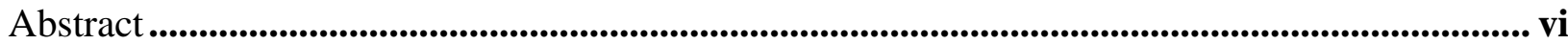

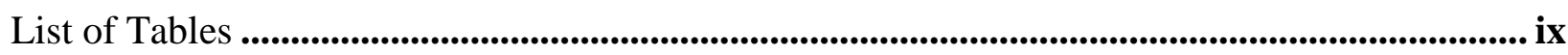

List of Figures .............................................................................................................................................. $\mathrm{x}$

Chapter 1. Introduction ............................................................................................................................... 1

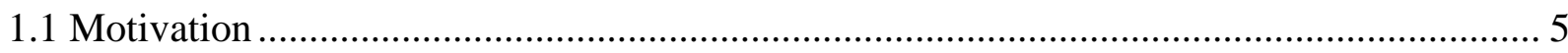

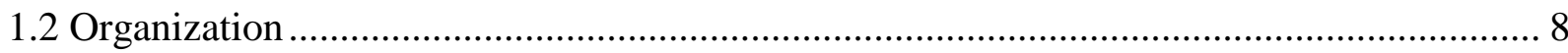

Chapter 2. Literature Review .................................................................................................................. 9

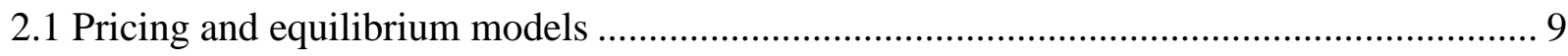

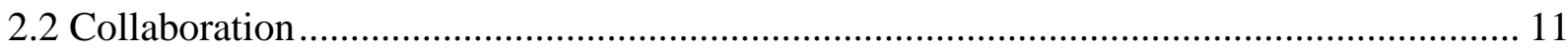

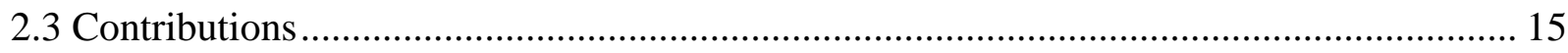

Chapter 3. Model Description and Formulation................................................................................... 16

Chapter 4. Solution Methodology......................................................................................................... 21

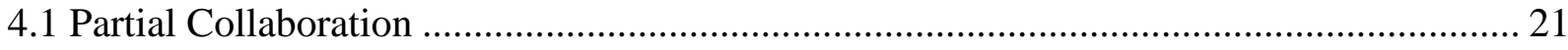

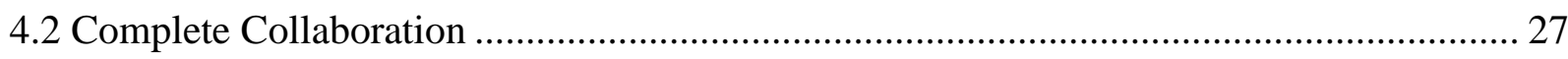

Chapter 5. Results and Discussion ................................................................................................... 32

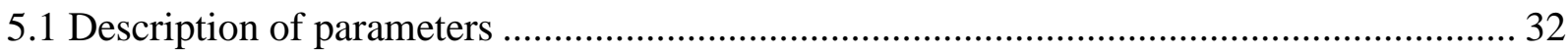

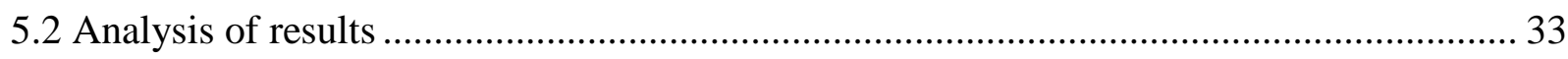

Chapter 6. Conclusions.............................................................................................................................. 42

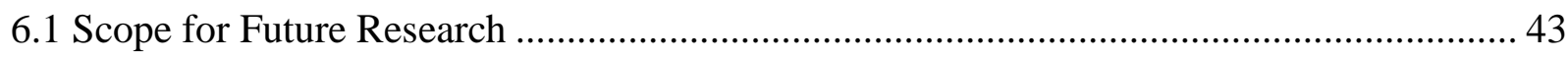

Appendices........................................................................................................................................... 45

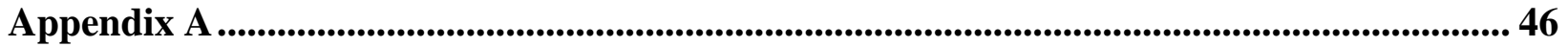

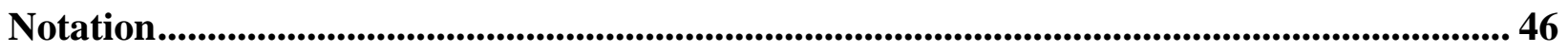

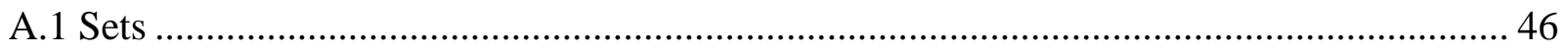

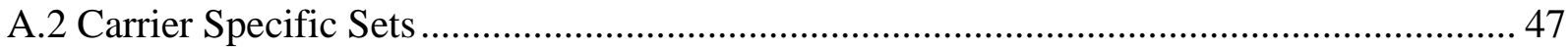

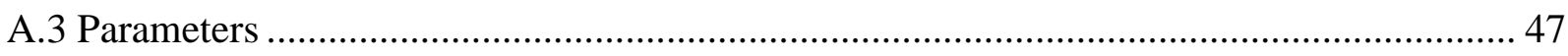

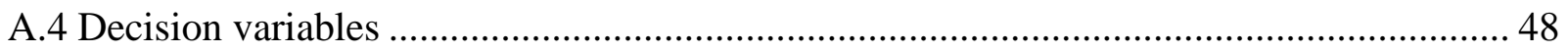




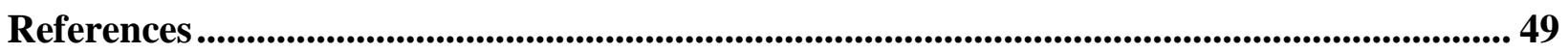

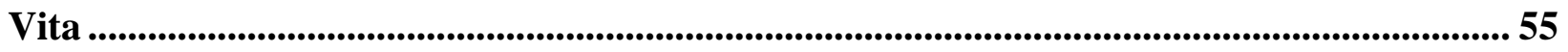




\section{List of Tables}

Table 1.1 National Expenditure on Freight Transportation ([7]) .......................................................

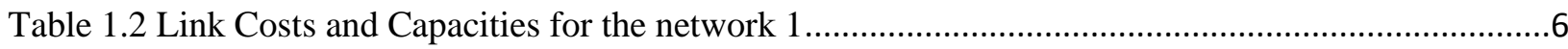

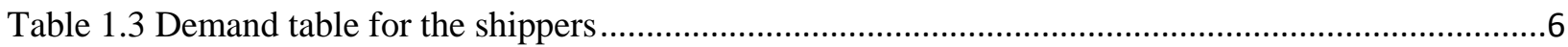

Table 5.1 Profit Comparison: 20 Node network, 2 carriers.............................................................. 34

Table 5.2 Profit Comparison: 30, 40 and 50 Node network, 2 carriers ................................................ 36

Table 5.3 Profit Comparison: 40 Node network, 3 carriers................................................................. 38

Table 5.4 Profit Comparison: 50 Node network, 3 carriers............................................................... 39

Table 5.5 Profit Comparisons: Complete Collaboration vs. Partial Collaboration for a 40 node

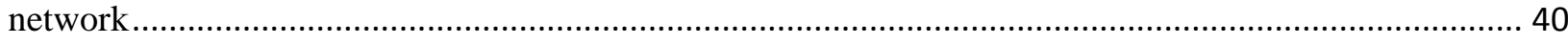

Table 5.6 Comparison of results for Shapley Value and Partial Collaboration........................................ 41 


\section{List of Figures}

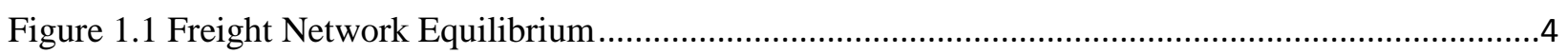

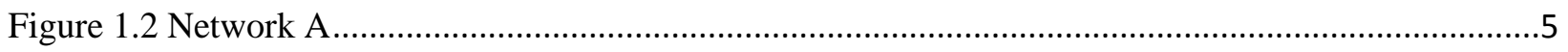

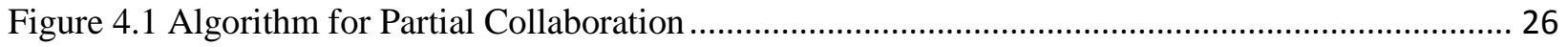

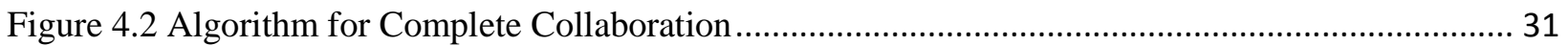




\section{Chapter 1}

\section{Introduction}

Freight transportation is at the core of the development and operation of human society. It plays a significant role in the smooth functioning of the economy by connecting and binding together the spatially distributed producers and consumers. Starting from assembling the raw material to the delivery of the final product to the consumer, freight transportation plays a vital role in every link of the supply chain. With the advent of globalization and new logistic practices such as just in time delivery services it has become even more important to develop efficient freight transportation systems. The importance of the freight transportation system can be underscored by the fact that around 17.5 billion metric tons of goods valued at over 13 trillion US dollars were transported in 2002. This volume is expected to double and reach 35 billion metric tons by 2035 [1]. The values of the expenditure on freight transportation as a percentage of gross national product of the countries mentioned in Table 1.1 further ascertain the need

to study freight transportation systems. Transportation expenditures also represent a significant part of the final price of the product. It has been estimated that this part may reach $13 \%$ for the primary industry sector and $11 \%$ for the transformation and production sector [31]. Given the volume, monetary value of the goods being transported and its 
impact on the economy it is essential to develop mathematical models to study the various agents impacting freight movement. One of the many other reasons to model freight transportation is to study its impact on other transportation systems such as road, rail and air networks.

Table 1.1 National Expenditure on Freight Transportation ([7])

\begin{tabular}{|c|c|}
\hline Country & $\begin{array}{c}\text { Expenditure (\% of } \\
\text { GNP) }\end{array}$ \\
\hline USA & $10 \%$ \\
\hline Canada & $16 \%$ \\
\hline United Kingdom & $15 \%$ \\
\hline
\end{tabular}

The freight transportation system is a very complex system involving multiple players with conflicting interests. The infrastructure decisions to be made are long term in nature and usually encounter heavy implementation delays. Furthermore, it has to adapt to the rapid changes arising due to social and political policy changes and trends in the economy. Thus it is essential to develop an efficient platform to analyze the planning, operations and decision making processes.

The freight transportation system derives its demand from the interplay between producers and consumers who are spatially separated. Producers require freight transportation to procure initial raw materials, move the intermediate products and to transfer the final products to the consumers. These transportation services are either managed by the producers themselves in which they act as the carriers as well or provided by external carriers. Finally, shippers are either the producers themselves or 
intermediary agents between the producers and consumers. Modeling the freight industry is a complicated process as the interest of a number of stakeholders with conflicting objectives need to be captured. One of the first works in this area was conducted by [13], [14] and [12], who used the spatial network equilibrium principle to capture interaction between producers, consumers and shippers. The two main stakeholders, the producers and the consumers interact with each other in the market based on the price of the commodity. Traditionally in economic models deterministic demand supply relationships are used to capture the interaction between producers and consumers. In most cases producers and consumers are spatially separated. This is becoming increasingly prevalent in a global economy where the producers and consumers are in different parts of the world. The producers contact the shippers who coordinate the transport of goods from the production facility to the consumer location. The shippers then contact carriers who actually facilitate the movement of goods through trucks, rail or ships. The shipper's objective is to transport the commodities to the destination at the minimum cost. On the other hand, the carriers seek to maximize their profit by attracting as much demand as possible to the sub-network under their control and also minimizing the cost of routing within their sub-network. This interaction results in a two player game involving a shipper and carrier/carriers. The shippers select a carrier or a combination of carriers, who control the transportation infrastructure, for each shipment based on the prices set by the carriers. Figure 1.1 represents the interaction between various players in the freight industry. The outcomes of the interactions between various agents in the freight market are indicated on the link connecting them in the diagram. 


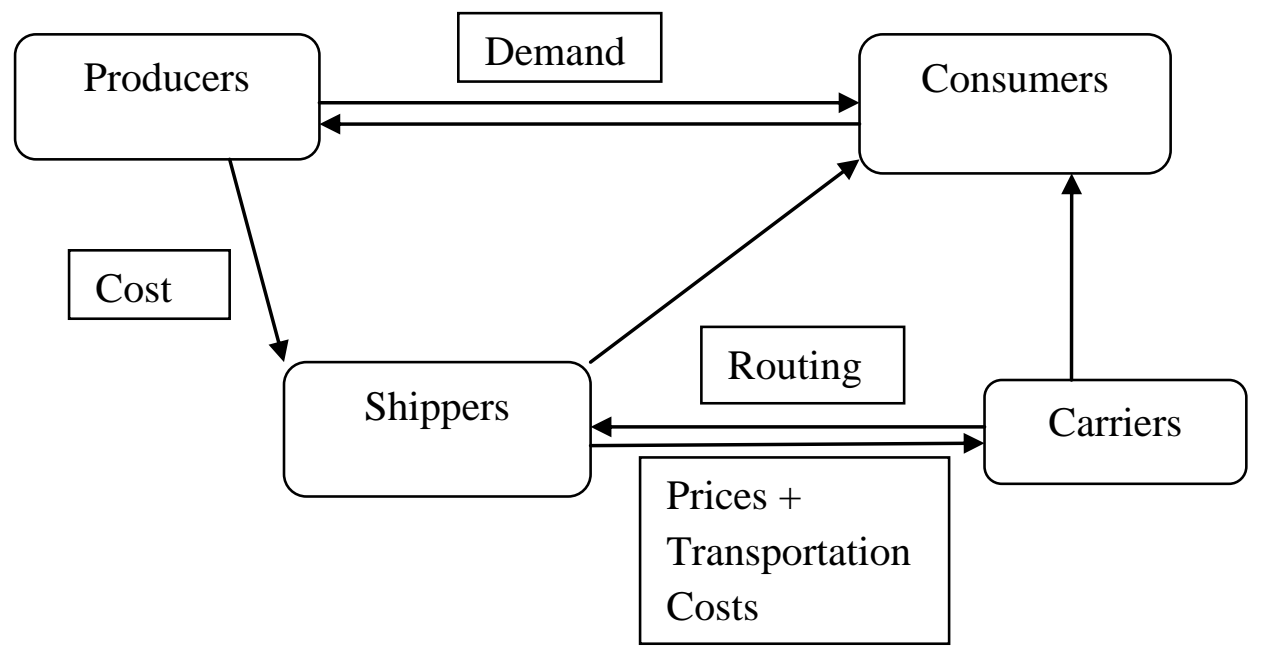

Figure 1.1 Freight Network Equilibrium

The magnitude of shipment attracted by a carrier depends upon the level of service it offers and the prices it enforces. The three dominant level of service parameters are the unit cost of transportation, the capacity and the shipment time. The carrier's ability to provide transportation services in turn would depend on the scale of services. Reference [19] developed an integrated model capturing the interaction between four main agents using the spatial network equilibrium formulation to model the demand side and the freight network equilibrium model to capture the interaction between shippers and carriers. Note that the shippers and carriers play an important role in determining the prices of commodities as their interaction determines the transportation cost which is a component of the final price of the commodity being transported. For example, transportation delays can at times increase the prices of the commodities by up to $250 \%$ [38]. This percentage is bound to increase when the goods being transported are perishable. Efficient interaction between carriers can lead to a significant savings in 
transportation costs and reduction in prices of commodities and improved profits for all the agents involved.

\subsection{Motivation}

As mentioned earlier the cost of transportation has a heavy impact on the final price of the product. The cost of transportation can be reduced if the carriers are somehow able to come up with new strategies that will result in efficiently transferring the goods between various origin-destination pairs. One of the common ways in which carriers reduce cost is by forming alliances for efficient utilization of the transportation assets under their

control. For example when a number of carriers pool their resources and form a collaboration they can achieve higher economies of scale enabling them to transport the goods at reduced costs and thus attracting more customers.

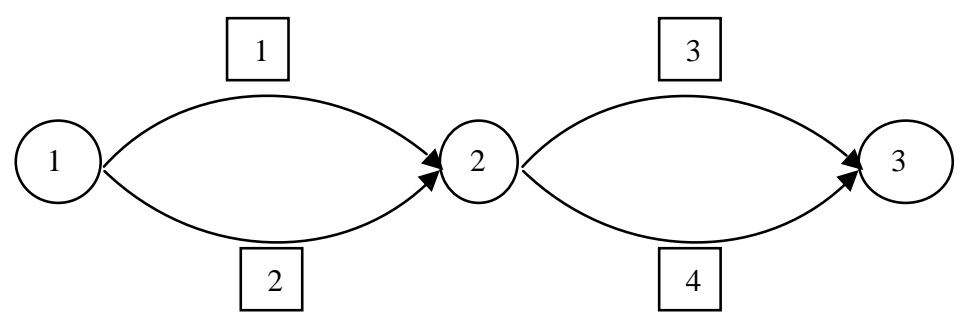

Figure 1.2 Network A

Consider the network shown in Figure 1.2 (Network A). Link 1and 3 are under the control of the lead carriers 1 and 2 respectively, while the links 2 and 4 are operated by the secondary carrier. The cost of transportation on the leader carrier arcs (does not 
include the prices imposed) and the secondary carrier arcs (overall cost) and the arc capacities are mentioned in Table 1.2.

Table 1.2 Link Costs and Capacities for the network 1

\begin{tabular}{|c|c|c|c|}
\hline Link & Cost & Capacity & $\begin{array}{c}\text { Controlling } \\
\text { Agent }\end{array}$ \\
\hline 1 & 2 & 20 & Lead Carrier 1 \\
\hline 2 & 4 & 100 & $\begin{array}{c}\text { Secondary } \\
\text { Carrier }\end{array}$ \\
\hline 3 & 2 & 20 & $\begin{array}{c}\text { Lead Carrier 2 } \\
\text { Secondary } \\
\text { Carrier }\end{array}$ \\
\hline 4
\end{tabular}

Table 1.3 Demand table for the shippers

\begin{tabular}{|c|c|c|c|}
\hline Shipper & Origin & Destination & Volume \\
\hline Shipper 1 & 1 & 3 & 10 units \\
\hline Shipper 2 & 1 & 3 & 10 units \\
\hline
\end{tabular}

Table 1.3 provides the demands to be satisfied by each shipper. Note that shipper 1 interacts only with carrier 1 and the secondary carriers, while shipper 2 interacts only with carrier 2 and secondary carriers. If the lead carriers were to act independently, i.e. with no sort of collaboration with the other lead carriers, then the profits gained by each of them would be 20 . But instead, if carrier 1 were to offer its residual capacity of 10 units on link 1 to carrier 2 and carrier 2 offers its residual capacity of 10 units on link 3 to carrier 1, their individual profits will increase by 20 respectively (assuming that the capacity of one carrier is offered at the transportation cost to the other carrier). Modeling 
this collaboration is not straightforward. A number of issues arise when the number of carriers trying to form the coalition in the market increases. Also, as the network size gets bigger the sharing of capacities gets complicated. Apart from the difficulties arising from the network structure and the problem size, the market lays a number of rules in forming the collaboration that are to be factored in before arriving at an optimal collaboration strategy.

However, in a coalition sometimes the coalitions' objectives may conflict with the objectives of the individual participants. In the carrier collaboration case, transportation resource utilization strategies which maximize the profit of the coalition may not maximize the profit of certain individual players. In such cases the coalition may not be sustainable. In this work we present two methodologies for modeling carrier collaboration in shipper carrier networks. The first one is a partial collaboration framework and the second methodology involves complete collaboration among carriers. The behavior of the carriers and shippers is assumed to be described by the bi-level noncooperative framework developed by [3]. In the model a shipper aims to transport a known amount of demand from sources to sink at minimum cost. The shipper has the option of choosing either the leader carrier or the set of secondary follower carriers. Whenever the shipper transports goods on a particular arc he gets charged unit transportation cost and the price set by the carrier. The leader carrier and secondary carrier each have control over a subset of arcs in the network. The leader carrier sets prices on his arcs as to maximize his profit. The secondary carrier is assumed not to vary 
his price. Such a structure can be used to model certain industries such as coal and a similar structure has also been found efficient in modeling revenue management for airlines [28].

In this paper the focus is on studying collaboration between multiple leader carriers, each of them competing for a demand against secondary follower carrier. For example let us assume there are two leader carriers LC1 and LC2. Leader Carrier LC1 sets price to maximize its profit when competing for a known demand D1 against secondary carriers. In the same way Leader Carrier LC2 sets prices to maximize its profit when competing for a known demand D2 against secondary carriers. Thus the two leader carriers have independent demands and they do not compete with each other. However, they do try to collaborate and help each other to efficiently utilize their resources against secondary carriers.

\subsection{Organization}

The paper is organized as follows. The next section provides an overview of a subset of past works on freight pricing and carrier collaboration. A description of the two models developed in this paper followed by the mathematical programming formulation and solution methodology is provided in section 3 and 4 . Section 5 presents the numerical analysis and compares the solutions of the two collaboration strategies to the case where carriers act individually. Some of the salient conclusions are presented followed by directions for future research. 


\section{Chapter 2}

\section{Literature Review}

The current research focuses on two different aspects of freight modeling: (a) the pricing of network and (b) carrier collaboration. The following sections provide a brief review of literature on pricing and carrier collaboration in freight networks.

\subsection{Pricing and equilibrium models}

Most of the early models reported in the literature which took into consideration both the shipper and carrier while modeling freight networks had the drawback of being oversimplified. For instance, in the sequential shipper-carrier model by [14], the shippers select the commodity origins and the carrier based on their perception of the transportation network, thus determining the demand, while the carriers respond to these demands by routing the freight over their portion of the network. The main drawback in this model was that the prices set by the carriers were assumed to be a fraction of the cost incurred by them and thus it is not a decision variable in the model. This model falls under the category of predictive models where the objective is to determine the allocation of freight flows among different carriers. Other such models include [17], [18], [19] and [11]. In one of the most recent works in this category, [21] developed a model where the shipper selects an output and a carrier or a coalition of carriers used for each shipment, 
given carrier price schedules. The principal difference between this model and previous models was the assumption about the form of carrier pricing. Instead of assuming that the carriers use constant prices, which may fail to be optimal and inconsistent with the usual practice ([20], [33] and [37]), the model allows carriers to choose prices which depend on the volume.

Reference [3] developed a bi-level model for the freight price setting problem. A key difference between this model and the previous models was the emphasis on the price side rather than on the allocation of freight flows among the carriers. Also, the problem addressed was for a single commodity flow, which is the case for lower level firms whose demand for transportation depends solely on the location of its supply and demand sites. The problem is modeled in a bi-level program setting where at the lower level the shippers tries to satisfy its demand at the lowest cost possible, given the price schedule. The resulting flow pattern is obtained by solving a transshipment problem where the prices are added to the initial arc costs. At the upper level, the carrier's objective is to maximize its revenues by setting optimal prices on the subset of arcs in its control. The carrier assumes no competition from other carriers but takes into consideration the reaction of the shipper to its price structure. In an extension to this model, [5] formulated a problem where the carrier not only has to set the prices on the arcs over which it has control, but also determined the capacity to be installed on each of the arcs. Along similar lines, [4] modeled a joint network design problem involving multi-commodity flow. In 
this case at the upper level the leader carrier is concerned with maximizing the profit raised from prices set on a subset of arcs which are also to be determined.

\subsection{Collaboration}

There have been many arguments over the years as to whether open communication and information sharing can exist between players with conflicting interests. Dan Goodwill, President of Dan Goodwill and Associates Inc, argues that shippers need to share as much data as possible with their carriers concerning their freight characteristics, volumes, lanes, delivery requirements and their freight rates. Sharing this information with existing and prospective core carriers is crucial since there is no point trying to establish a collaborative relationship with companies with whom your freight characteristics are not a fit. Similarly, it is essential that carriers share their strengths in terms of head haul and back haul requirements, capacity and service levels so that a good relation exists with its customers. As situations change (e.g. new customers are added, the carrier's head haul and back haul requirements become different), it is important to share this information with each other to sustain the relationship. Data from a recent study suggests that shippers that do work in a collaborative rather than adversarial role with their carriers tend to spend less on freight as a percent of revenue. Collaboration is a challenge but it is in the best interests of both shippers and carriers [15].

The area of carrier collaboration is increasingly receiving attention with the advent of latest information, communication and database technologies which have updated online information on the usage of various transportation assets. A variety of economic reasons 
exist for the formation of inter-organizational collaboration. These include sharing the costs of large investments, achieving economies of scale, better utilization of expensive assets, pooling and spreading risk, and gaining access to complementary resources [16]. Additionally, increasing the return on investment (ROI) by geographically widening the marketplace for a firm's products and services can be a motivator of collaborative relationships. Overall, literature on collaboration at inter-organizational level has developed a number of theoretical arguments to explain the formation and structure of alliances between organizations [8]. Reference [8] conducted a descriptive study on the impact of collaborative transportation management (CTM) on shippers and carriers and found that collaborating and utilizing combined resources can lead to significant benefits for all participants. In general applying CTM principles was found to significantly reduce transportation cost, improve pick-up and delivery on time performance which is very critical for just in time delivery practices, better asset utilization by reduction of zero load miles on fleets and dwell times and significant reduction in administrative lead time needed to plan and execute various transportation activities. Reference [30] developed mathematical models to quantify the social benefit of consolidation of urban freight carriers. The results from the study reveal that consolidation of freight carriers in an urban area can cause significant reduction in congestion by reducing the flow of trucks on the street. The paper also stressed that for consolidation to be successful numerous policies should be enacted by the government to provide incentives for carriers to collaborate. Reference [22] discusses six business models for transportation collaboration and shows that significant cost savings can be achieved. 
Many studies have focused on carrier collaboration strategies in an auction setting applying principles of auction theory and game theoretic concepts [25]. Reference [29] studied rational allocation mechanisms for bargaining problems between one buyer and seller and proved the non-existence of efficient mechanisms without subsidies. Reference [35] proposes an auction based collaborative mechanisms for carriers with service areas which overlap. In this mechanism whenever a carrier gets a load which is not cost effective to serve he invites bids from other carriers and conducts a second price auction. Reference [9] develop a dynamic collaborative mechanism framework for studying carrier interaction in a transportation auction market place where each carrier receives a random and time dependent demand. Whenever a carrier receives a new shipment demand, an incentive is provided for the carrier to submit the request to the collaborative mechanism. A simulation based analysis of multiple carriers engaging in truckload pickup and delivery in overlapping service areas shows that the collaborative mechanism is more efficient than the non-collaborative case. Reference [10] apply distributed artificial intelligence techniques to develop a multi-agent based simulation platform for studying collaboration among shipping companies with focus on decommittment strategy which may not be acceptable in the freight marketplace [9]. Reference [24] applies auction and game theory principles to develop incentives to encourage freight forwarders to collaborate in a combinatorial auction setting. However, the profit sharing mechanism developed in this work assumes that the market forces affecting all participants are similar which may not be the case. 
Reference [26] adopts a mathematical programming approach to study collaborative planning for container vehicle scheduling and compares the performance against two non-collaborative strategies. The results show that if all the participants in the coalition are committed to sharing resources and demand, then collaborative strategies significantly outperform other strategies. Previously, companies focused on maximizing their own profits using the assets available at their disposal. However recently the many and varied carriers, who in the past worked independently of each other, are working in close liaison. The sea-cargo industry is no exception. Since 1990, when Sea-Land and Maersk introduced the alliance system and began sharing vessels in the Atlantic and Pacific oceans, mergers have become increasingly common. Recently, smaller alliances are collaborating to form even bigger alliances, for example The Grand Alliance and The New World Alliance laid down foundations for cooperating in 2006 [1]. References [1] and [2] develop a mechanism based on mathematical programming and game theory to determine incentives to encourage liner ship operators to participate and form alliances. The objective of the incentive is to encourage operators to act in a way which maximizes the entire alliance benefits while maximizing the individual profits.

Reference [32] addresses the issue of high competition contracts from large organizations that often prefer a relationship with only one transport provider. The paper argues that joining forces to form a road haulage consortium can allow small-to medium operators to compete against larger players by improving services to the customer through reduced delivery times and wider geographical coverage. Collaboration has potential benefits not 
only for freight transport service providers in terms of efficiency and utilization, but also for the wider society through increased quality of life.

\subsection{Contributions}

1) Two heuristics are developed to model the carrier collaboration in freight networks. To the best of our knowledge we are not aware of any prior works where pricing and collaboration are modeled together. Our model allows the carriers to price the part of the network under its control and at the same time determines the capacity it is willing to share with other carriers.

2) Principles from cooperative game theory (Shapley Value) are used to model collaboration in one of the heuristics.

3) The heuristics were applied to networks of varying sizes for different demand values. The results supported the argument that collaboration results in improved profits for every agent involved in the collaboration.

This work studies the impact of carrier collaboration on carriers pricing decision. Two types of collaboration are studied: partial and complete. The models apply principles of mathematical programming and cooperative game theory. When carriers collaborate and efficiently utilize their resources then they can price their commodities efficiently leading to more demand and increased profit. The next section describes the mathematical program used by a single carrier to determine the price on the arcs. 


\section{Chapter 3}

\section{Model Description and Formulation}

As mentioned earlier the price setting problem for a single carrier used in this work is the capacitated version of the price setting problem extensively studied by [3],[4],[5] and [27]. Consider a network $G=(N, A)$ where $N$ denotes the set of nodes and $A$ represents the set of arcs. In this network the shipper wants to transport goods from the sources (producer nodes) to the sinks (consumer nodes) at minimum cost. $O$ represents the set of source nodes and $D$ represents the set of sink nodes. The arcs in the network are either under the control of a leader carrier or under the control of a collection of secondary follower carriers who operate as one carrier. Let $A_{1}$ represent the arcs in the network under the control of the leader carrier and $A_{2}$ represent the arcs in the network under the control of the secondary follower carrier. Whenever a shipper uses an arc the shipper is charged a cost comprising the transportation cost and a price levied by the carrier. The secondary follower carrier charges a unit price $d_{a} \forall a \epsilon A_{2}$ comprising the price and transportation cost. The leader carrier charges a transportation cost $c_{a}$ and a price $\mathrm{p}_{\mathrm{a}}$ for all arcs in the set $A_{1}$. The objective of this problem is to determine the price to be levied by the leader carrier to maximize his profit. The problem can be formulated as a bi-level mathematical program as follows: 


\section{BILEVEL BILINEAR}

$$
\begin{gathered}
\max \sum_{a \epsilon A_{1}} p_{a} x_{a} \\
\min \sum_{a \epsilon A_{1}}\left(c_{a}+p_{a}\right) x_{a}+\sum_{a \epsilon A_{2}} d_{a} y_{a} \\
\sum_{a \epsilon \Gamma^{-1}(i) \cap A_{1}} x_{a}+\sum_{a \epsilon \Gamma^{-1}(i) \cap A_{2}} y_{a}-\sum_{a \epsilon \Gamma(i) \cap A_{1}} x_{a}-\sum_{a \epsilon \Gamma(i) \cap A_{2}} y_{a}=b_{i} \forall i \epsilon N \\
x_{a} \leq \operatorname{cap}_{a} \forall a \epsilon A_{1} \\
x_{a} \geq 0, y_{a} \geq 0
\end{gathered}
$$

In the above formulation $x_{a}$ denotes the flow on leader carrier arcs $A_{1}$ and $y_{a}$ denotes the flow on secondary follower carrier arcs. $\Gamma^{-1}(i)$ represents the set of arcs coming into node $i$ and $\Gamma(i)$ represents the set of arcs going out of node $i$. In the upper level the leader carrier sets prices on arcs under his control to maximize his profits. In the lower level, the shipper solves a minimum cost flow transshipment problem to transport the goods from the sources to the sinks. The above bi-level problem can be converted into a single level problem by dualizing the lower level problem and writing the primal dual optimality constraints as follows: 


\section{BILIN}

$$
\begin{gathered}
\max \sum_{a \epsilon A_{1}} p_{a} x_{a} \\
\sum_{a \epsilon \Gamma^{-1}(i) \cap A_{1}} x_{a}+\sum_{a \epsilon \Gamma^{-1}(i) \cap A_{2}} y_{a}-\sum_{a \epsilon \Gamma(i) \cap A_{1}} x_{a}-\sum_{a \epsilon \Gamma(i) \cap A_{2}} y_{a}=b_{i} \forall i \epsilon N \\
\lambda_{u_{a}}-\lambda_{v_{a}}+\delta_{a} \leq c_{a}+p_{a} \forall a \epsilon A_{1} \\
\lambda_{u_{a}}-\lambda_{v_{a}} \leq d_{a} \forall a \epsilon A_{2} \\
\sum_{a \epsilon A_{1}}\left(c_{a}+p_{a}\right) x_{a}+\sum_{a \epsilon A_{2}} d_{a} y_{a}=\sum_{i \epsilon N} b_{i} \lambda_{i}+\sum_{a \epsilon A_{1}} \delta_{a} c_{a} \\
x_{a} \leq \operatorname{cap}_{a} \forall a \epsilon A_{1} \\
x_{a} \geq 0, y_{a} \geq 0
\end{gathered}
$$

In the above problem $u_{a}$ and $v_{a}$ represent the upstream and downstream nodes of arc a. Primal dual penalty based heuristics have been studied for solving the above problem in [6] and [3]. However in this paper, the above problem is solved by rewriting the flow variables as a sum of binary variables and solving the resulting integer program. The process has been explained in detail in [3]. The resulting single level mixed integer programming formulation is as follows: 
MIP

$$
\begin{aligned}
& \underbrace{\max }_{p, y, z, \lambda} \sum_{a \in A_{1}} \sum_{k=0}^{\bar{k}} 2^{k} p_{a}^{k} \\
& \sum_{a \in \Gamma^{-1}(i) \cap} \sum_{A_{1}}^{k} 2^{k} z_{a}^{k}+\sum_{a \in \Gamma(i) \cap A_{1}} y_{a}-\sum_{a \in \Gamma^{-1}(i) \cap} \sum_{A_{2}}^{k} 2^{k} z_{a}^{k}-\sum_{a \in \Gamma(i) \cap A_{2}} y_{a} \\
& =b_{i} \forall i \in N \\
& \lambda_{u_{a}}-\lambda_{v_{a}}+\delta_{a} \leq c_{a}+p_{a} \forall a \in A_{1} \\
& \lambda_{u_{a}}-\lambda_{v_{a}} \leq d_{a} \forall a \in A_{2} \\
& \sum_{a \epsilon A_{1}}\left(c_{a} \sum_{k=0}^{k} 2^{k} z_{a}^{k}+\sum_{k=0}^{k} 2^{k} p_{a}^{k}\right)+\sum_{a \epsilon A_{2}} d_{a} y_{a}=\sum_{i \epsilon N} b_{i} \lambda_{i}+\sum_{a \epsilon A_{1}} \delta_{a} c_{a} \\
& -M z_{a}^{k} \leq p_{a}^{k} \leq M z_{a}^{k} \forall a \epsilon A_{1} \forall k \in K \\
& -M\left(1-z_{a}^{k}\right) \leq p_{a}^{k}-p_{a} \leq M\left(1-z_{a}^{k}\right) \forall a \epsilon A_{1} \forall k \in K \\
& \sum_{k=0}^{k} 2^{k} z_{a}^{k} \leq \operatorname{cap}_{a} \forall a \in A_{1} \\
& z_{a}^{k} \in\{0,1\} a \in A_{1} \forall k \in K \\
& y_{a} \geq 0, \delta_{a} \leq 0
\end{aligned}
$$

The MIP formulation is obtained by performing the following transformation to the flow variables:

$$
x_{a}=\sum_{k=0}^{\bar{k}} 2^{k} z_{a}^{k}
$$




$$
\begin{gathered}
\bar{k}=\left\lfloor\log _{2}\left(\sum_{i \in N: b_{i}>0}\left(b_{i}+1\right)\right)\right\rfloor \\
z_{a}^{k} \in\{0,1\} \forall k \quad \ni 0 \leq k \leq k
\end{gathered}
$$

The flow can be expressed in this form as it is bounded above by the total demand in the system. Also, the representation of a flow as a sum of binary variables can be justified by the following lemma:

Lemma 3.1: Every positive integer can also be uniquely represented as a sum of different powers of 2 .

This section provided the description and formulation of the basic freight price setting model. This model will be used extensively throughout the solution methodology for the two collaboration strategies described in the following section. 


\section{Chapter 4}

\section{Solution Methodology}

The two carrier collaboration models developed are explained in this section. One should note that the collaboration studied in this work is alliance formation between the leader carriers. The first one is partial cooperation where each carrier offers the residual capacity of the arcs under its control to the other carrier. The carriers then resolve the price setting problem with the new network and accept the proposal only if the proposal increases their profit. The second carrier collaboration principle is based on principles of cooperative game theory where all the carriers collaborate completely and solve the price setting problem as one single carrier. Then using the principle of the Shapley Value the profits are distributed among the leader carriers. Section 4.1 presents the partial collaboration models. The complete collaboration based model is explained in section 4.2.

\subsection{Partial Collaboration}

This section explains the methodology used for modeling partial collaboration among the leader carriers in the network. Consider a network $G=(N, A)$ where $N$ denotes the set of nodes and $A$ denotes the set of arcs. There are $\mathrm{M}$ shippers operating in this network attempting to transport goods from source nodes $O^{m}$ to destination nodes $D^{m}$ at 
minimum cost. $O^{m}$ and $D^{m}$ represent source and sink nodes for shipper $m$. There are $M$ leader carriers operating in this network. Let $A_{1}^{m}$ represent the set of arcs under the control of leader carrier $m$ and $A_{2}$ represent the set of arcs under the control of the secondary follower carrier. Note that the sets $A_{1}^{m}, \forall m=1, \ldots, M$ and $A_{2}$ are disjoint sets, i.e., $A_{1}^{1} \cap A_{1}^{2} \cap A_{1}^{3} \ldots \cap A_{1}^{M} \cap A_{2}=\emptyset$. Each leader carrier charges the shipper a unit price $p_{a}^{m}$ and a unit transportation cost $c_{a}^{m}$ for using the arcs under his control. The leader carrier arcs are capacitated. The secondary follower carrier charges the shipper a unit cost $d^{a}$ for using the arcs. Each shipper $m$ is assumed to interact with the leader carrier $m$ and the secondary follower carrier for transporting goods from source nodes $O^{m}$ to destination nodes $D^{m}$. Let $b^{m}$ represent the vector of goods to be transported. If there is no carrier collaboration the leader carrier $m$ sets prices on arcs under his control to maximize his profit applying the mathematical program defined in section 3. Once the leader carrier sets his prices the shipper $m$ is aware of the costs of transport on every arc in the network comprised by arcs $A_{1}^{m} \cup A_{2}$ and chooses a minimum cost routing strategy for the demand vector bm. Note that the shippers are not assumed to interact with each other. In the case where there is no carrier collaboration, each carrier m individually determines its price to maximize its profit while competing against secondary carriers. Also the leader carriers are assumed to compete only against the secondary carrier and not with each other, i.e., each leader carrier $m$ interacts with shipper $m$ for a demand $b^{m}$. 
However, many leader carriers may have arcs with unused capacity. If the leader carriers collaborate there is potential for each carrier to use each others' unused capacity and increase their profit by providing a more efficient and cheaper mode of transport to the shipper. Unused capacity is extremely common in less than truckload (LTL) delivery systems which constitutes a significant portion of freight transport in the US. If the unused capacity can be used this can result in significant cost savings especially with increasing fuel prices. In this section a partial collaboration model is presented to model sharing of capacity between leader carriers.

In the partial collaboration model, carrier 1 first solves the price setting problem using the mathematical program explained earlier. The shipper under consideration is shipper 1 who is attempting to transport the demand $b^{1}$ to the respective destinations at minimum cost. The network under consideration is $A_{1}^{1} \cup A_{2}$. Once the leader carrier determines the optimal price and the shipper determines the routing strategy, the leader carrier 1 identifies arcs under its control with residual capacities, $A_{1}^{1^{\prime}} \in A_{1}^{1}$. The arcs with residual capacities are then offered to the second leader carrier at a price equal to the cost of transportation of goods on the respective arcs. Leader carrier 2 then solves his price setting problem with shipper 2 to transport demand $b^{2}$. The leader carrier 2 now operates on the arcs under his control $A_{1}^{2}$ along with arcs of leader carrier 1 with residual capacity $A_{1}^{1^{\prime}}$ (the available operating capacity is equal to the residual capacity obtained from solving the carrier 1 price setting problem). Note that when shipper 2 uses the arcs 
with residual capacity, $A_{1}^{1^{\prime}}$ belonging to carrier 1 he is charged an amount equal to transportation cost and leader carrier 2 price. Note that the profit obtained by leader carrier 2 when using $A_{1}^{2} \cup A_{1}^{1^{\prime}}$ is bound to be greater than the profit obtained by the leader carrier 2 when he uses only $A_{1}^{2}$. This is because the optimal solution to the price setting problem solved by leader carrier 2 on arcs under his control is a feasible solution to the price setting problem solved by leader carrier 2 when he uses the $\operatorname{arcs} A_{1}^{2} \cup A_{1}^{1^{\prime}}$. Leader carrier 2 determines the arcs under his control which have residual capacity $A_{1}^{2^{\prime}} \in A_{1}^{2}$ and offers it to the rest of the carriers. Now carrier 3 solves his price setting problem using arcs under his control $A_{1}^{3}$, the arcs under the control of leader carrier 2 with residual capacity $A_{1}^{2^{\prime}}$ and the arcs under the control of leader carrier 1 with residual capacity $A_{1}^{1^{\prime \prime}}$ after being used by leader carrier 1 and leader carrier 2 . Note that if shipper 3 uses arcs in $A_{1}^{2^{\prime}}$ and $A_{1}^{1^{\prime \prime}}$ he is charged the transportation cost and the price charged by carrier 3. Once all $m$ carriers have solved their problem, carrier 1 once again resolves his price setting problem on the network comprising arcs under his control and the residual capacity of arcs under the control of all other carriers. This process is completed when using other carriers' arcs does not increase any carriers' profit, i.e., equilibrium is reached as there is no incentive for any leader carrier to change his strategy. The procedure is represented on a flowchart in Figure 4.1.

Note that even though the above methodology is a heuristic it is similar in intuition to the diagonalization algorithm used to determine Cournot-Nash equilibria in the spatially 
separated network oligopoly problem [27]. In the diagonalization algorithm every player determines his most optimal strategy by fixing the strategies of all other players. The process continues till there is no incentive available for any player to improve his objective by changing his strategy. The methodology described in this section is called a partial collaboration strategy as every leader carrier solves only his strategy. The next section describes a complete collaboration strategy applying principles of cooperative game theory where all leader carriers join together, solve a system optimal problem and then distribute the profits. 


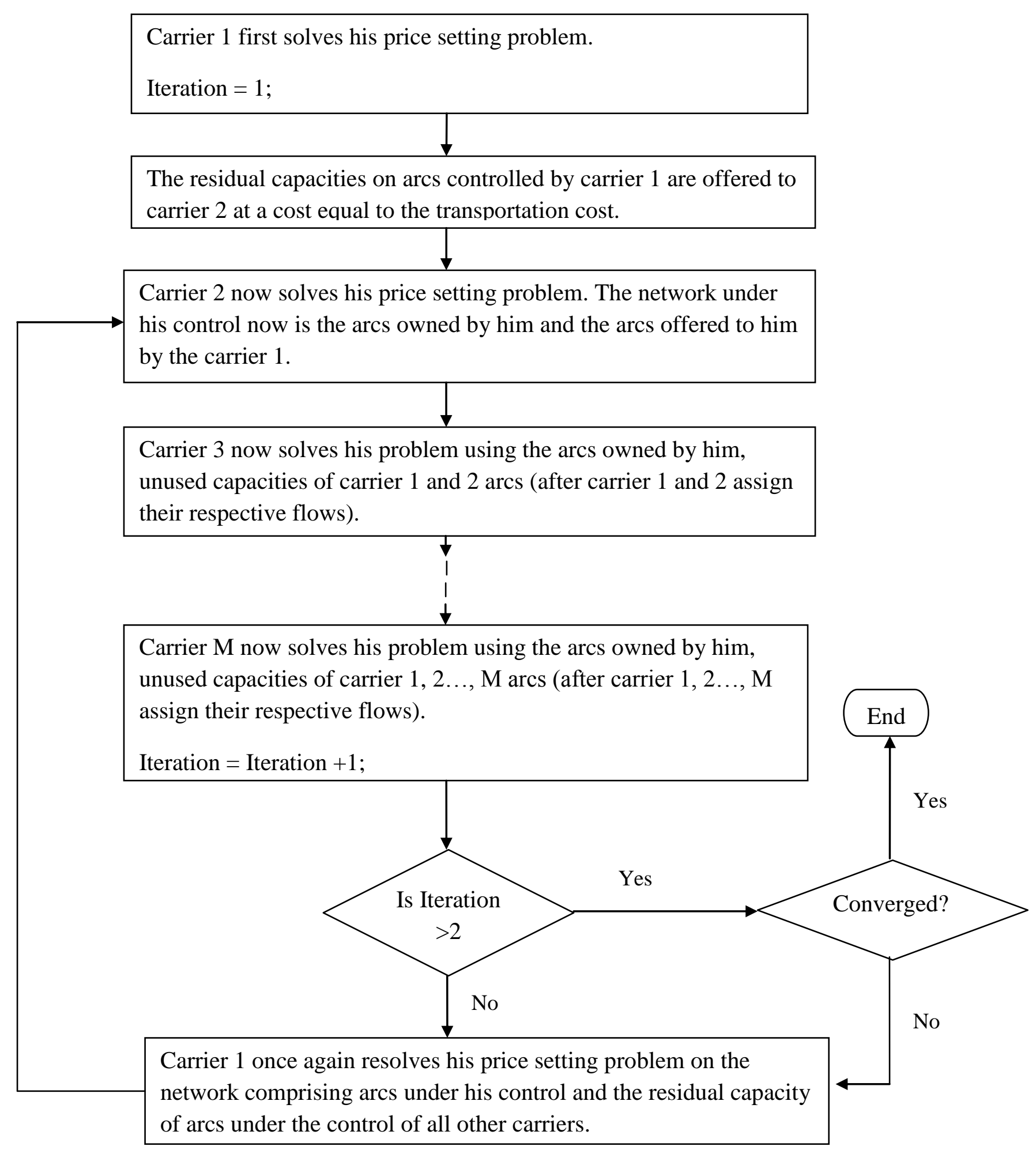

Figure 4.1 Algorithm for Partial Collaboration 


\subsection{Complete Collaboration}

This section describes a collaboration strategy developed based on principles of cooperative game theory. In cooperative game theory a group of players decides to form an alliance to maximize a joint objective. Examples of joint objectives could be to increase joint profit through increased economies of scale or higher market share. Note that each member of the alliance can obtain different benefits. However, the alliance or coalition will be sustainable only if the benefit obtained by each member by participating in the alliance exceeds the benefits obtained by each member acting independently. Thus, the distribution of the joint profits among the various participating members is the crucial part of modeling this collaboration strategy.

For the problem under study we assumed complete collaboration in which all leader carriers join together and form an alliance and act as one single leader carrier. This coalition of leader carriers then competes against the set of secondary follower carriers for the combined demand of all the shippers. So in this case the price setting problem is solved by assuming the leader carrier arcs to be the union of all individual leader carrier arcs, $A_{1}=A_{1}^{1} \cup A_{1}^{2} . . \cup A_{1}^{M}$. The joint profits are then distributed such that profit for each individual leader carrier obtained from participating in the coalition is greater than the profit obtained for each carrier when acting independently.

One of the common ways in which this joint benefit is shared among the members of the coalition is by using the Shapely Value [34] that distributes the joint profit based on the 
expected marginal contribution made by an individual to the coalition. For the above cooperative game the number of players is $M$. Let $C$ denote the set of coalitions and $S \in C$ represent a particular coalition. For any coalition we define a characteristic function $P(\mathrm{~S})$ which assigns a real number to any coalition $S \in C$. In this work $P(\mathrm{~S})$ refers to the joint profit obtained by the coalition S. For any individual leader carrier $i$ the Shapely Value is defined as:

$$
P S_{i}=\sum_{S \in M, i \in S} \frac{(|S|-1) !(M-|S|) !}{M !}[P(S)-P(S-i)]
$$

where, $|S|$ represents the number of members in coalition $S . S-i$ refers to the coalition minus member $i$. If leader carrier $i$ joins the coalition $S-i$ then $i$ receives the marginal profit $P(S)-P(S-i)$. The probability of carrier $i$ joining the coalition is $\frac{(|S|-1) !(M-|S|) !}{M !}$. Therefore, the expected marginal contribution is calculated as the product of the marginal contribution across all possible coalitions and the probability of carrier $i$ being in that coalition.

The principle of the Shapley Value can be illustrated by a simple example.

Example 4.1: Suppose there are three carriers (A, B, C) operating on the network. The following are the individual profits obtained by each of them if they were to act independently: 


$$
P(A)=25 ; P(B)=31 ; P(C)=32
$$

The profits obtained by a coalition of two of the carriers are as follows:

$$
P(A B)=70 ; \quad P(A C)=57 ; P(B C)=79
$$

If all the carriers were to collaborate with one another the total profit gained by the coalition is equal to 100 units.

From the Shapley Value equation the profit share of carrier $A$ if a coalition is formed between three of the carriers is as follows:

$$
P S_{A}=\sum_{S \in M, i \in S} \frac{(|S|-1) !(M-|S|) !}{M !}[P(S)-P(S-i)]
$$

For carrier $A$, the set of coalitions involving carrier $A$ are $\{\mathrm{A}\},\{\mathrm{AB}\},\{\mathrm{AC}\},\{\mathrm{ABC}\}$ :

$$
\begin{aligned}
& P S_{A}=\frac{(1-1) !(3-1) !}{3 !}[P(A)-P(0)]+\frac{(2-1) !(3-2) !}{3 !}[P(A B)-P(B)] \\
& +\frac{(2-1) !(3-2) !}{3 !}[P(A C)-P(C)] \\
& +\frac{(3-1) !(3-3) !}{3 !}[P(A B C)-P(B C)] \\
& =1 / 3[25]+1 / 6[70-31]+1 / 6[57-32]+1 / 3[100-79] \\
& =26
\end{aligned}
$$


For carrier $B$, the set of coalitions involving carrier $B$ are $\{\mathrm{B}\},\{\mathrm{AB}\},\{\mathrm{BC}\},\{\mathrm{ABC}\}$ :

$$
\begin{aligned}
& P S_{B}=\frac{(1-1) !(3-1) !}{3 !}[P(B)-P(0)]+\frac{(2-1) !(3-2) !}{3 !}[P(A B)-P(A)] \\
& +\frac{(2-1) !(3-2) !}{3 !}[P(B C)-P(C)] \\
& +\frac{(3-1) !(3-3) !}{3 !}[P(A B C)-P(A C)] \\
& =1 / 3[31]+1 / 6[70-25]+1 / 6[79-32]+1 / 3[100-57] \\
& =40
\end{aligned}
$$

For carrier $C$, the set of coalitions involving carrier $C$ are $\{C\},\{\mathrm{AC}\},\{\mathrm{BC}\},\{\mathrm{ABC}\}$ :

$$
\begin{aligned}
& P S_{C}=\frac{(1-1) !(3-1) !}{3 !}[P(C)-P(0)]+\frac{(2-1) !(3-2) !}{3 !}[P(A C)-P(A)] \\
& +\frac{(2-1) !(3-2) !}{3 !}[P(B C)-P(B)] \\
& +\frac{(3-1) !(3-3) !}{3 !}[P(A B C)-P(A B)] \\
& =1 / 3[32]+1 / 6[57-25]+1 / 6[79-31]+1 / 3[100-70] \\
& =34
\end{aligned}
$$


It is evident from the above example that the profit gained by any carrier forming a coalition is always greater than or equal to the profit obtained under no coalition. The next section provides some of the numerical results obtained from the test runs performed on sample networks.

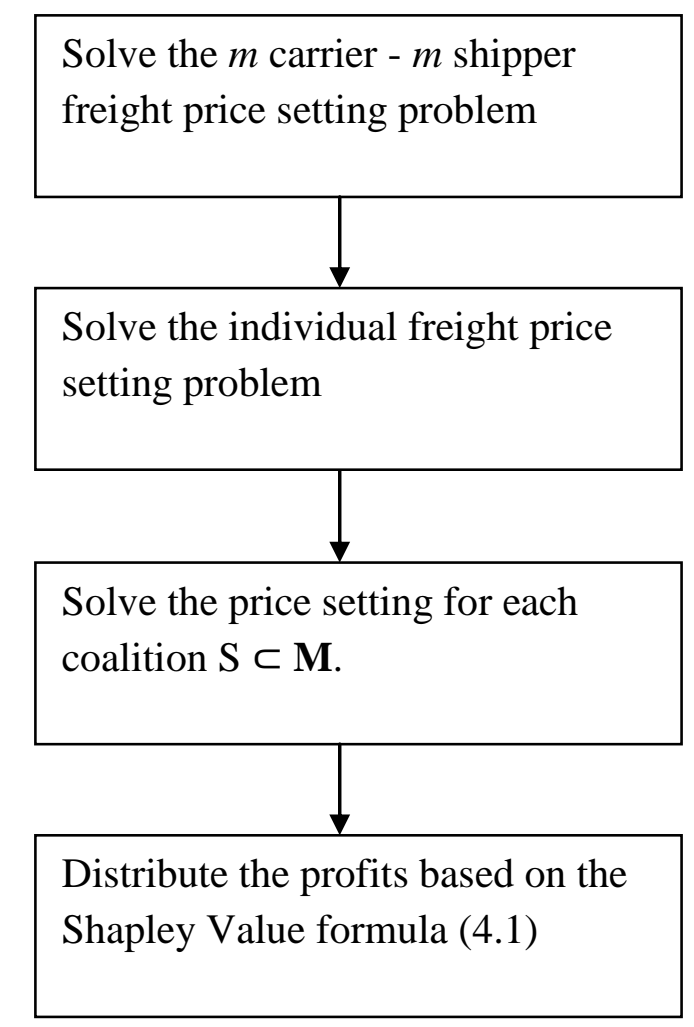

Figure 4.2 Algorithm for Complete Collaboration 


\section{Chapter 5}

\section{Results and Discussion}

The focus of the computational runs was to study the profit obtained through the two collaboration mechanisms proposed in this paper and compare it with the no collaboration scenario for various network topologies and demand levels. This section first presents an overview of the computational runs and then analyzes the results.

\subsection{Description of parameters}

The three collaboration mechanisms: individual price setting problem, partial collaboration and complete collaboration were implemented on a Windows HP machine with 3.4 GHz Pentium core two duo processor and $2 \mathrm{~GB}$ of RAM. The random network was created using NETGEN generator developed by [23]. The unit cost of transportation on an arc was assumed to vary between 4 and 6 for the lead carriers, while it is fixed at 20 for the secondary carriers. The capacities on the links for the lead carrier are fixed at 10 units. Four random networks with the following characteristics were generated using NETGEN: (i) 20 nodes and 50 arcs, (ii) 30 nodes and 121 arcs (iii) 40 nodes and 141 
arcs, and (iv) 50 nodes and 150 arcs. Five sets of demand values, very low, low, medium, high and very high, are generated for each network and for each carrier. The medium demand values are set to be equal to the feasible demands generated by [23]. All other demand levels were obtained by applying scaling factors to the medium demand, ranging from 0.5 to 3.0. The CPLEX solver in GAMS was used to solve all the three collaboration models.

\subsection{Analysis of results}

This section analyzes and compares the result obtained for the three collaboration mechanisms: (1) No collaboration between carriers, (2) Partial collaboration between carriers and (3) Complete collaboration between carriers and shippers. A detailed interpretation is provided for all the results obtained. The initial set of results compares the different collaboration strategies for a two carrier case followed by the three carrier collaboration results. Numerical results are also provided for multiple demand scenarios.

Table 5.1 provided the results for the complete collaboration, partial collaboration and no collaboration for the 20 node network with two leader carriers. The numbers in the table indicate the profit values. $\mathrm{C} 1$ and $\mathrm{C} 2$ refer to the two carriers and $\mathrm{C} 1$ (Ind) denotes the profit obtained by leader carrier $\mathrm{C} 1$ when he acts individually and does not collaborate with $\mathrm{C} 2$. The results for a 20 node network (Table 5.1) suggest that the complete 
collaboration may not always result in better profits compared to partial collaboration. In certain cases, the profit obtained from complete collaboration is equal to the total profit from partial collaboration. This situation usually arises when there is no competition for a common link between the two carriers.

Table 5.1 Profit Comparison: 20 Node network, 2 carriers

\begin{tabular}{|c|c|c|c|c|c|c|}
\hline Demand & $\begin{array}{c}\text { C1 } \\
\text { (Ind) }\end{array}$ & $\begin{array}{c}\text { C2 } \\
\text { (Ind) }\end{array}$ & \multicolumn{3}{|c|}{ C1+C2(Partial) } & C1+C2(Complete) \\
\hline & & & C1 & C2 & Total & \\
\hline Very Low & 120 & 100 & 200 & 180 & 380 & 380 \\
\hline Low & 120 & 120 & 200 & 240 & 440 & 440 \\
\hline Medium & 120 & 120 & 200 & 244 & 444 & 444 \\
\hline High & 120 & 120 & 200 & 252 & 452 & 452 \\
\hline Very High & 120 & 120 & 200 & 260 & 460 & 460 \\
\hline
\end{tabular}

The profit comparison for the three collaboration mechanisms for 30,40 and 50 node networks with 2 leader carriers operating on the network is shown in Table 5.2. The results for the partial collaboration and no collaboration indicate, as expected, that partial collaboration always provides profits higher than or equal to those obtained from no collaboration for each leader carrier. This increase in profit can be up to $50 \%$ for a carrier. In certain cases, for example the 40 node network at medium or higher demand levels, the profits obtained by a leader carrier (carrier 1 in this case) in the no collaboration scenario and partial collaboration scenarios might be equal. In such a 
situation, the leader carrier receiving no additional benefit from partial collaboration, carrier 1, might not form an alliance with the other leader carrier, carrier 2. The results for the complete collaboration scenario and partial collaboration scenario indicate that complete collaboration always gives a better or in the worst case the same profit as the partial collaboration. The profit being compared here is the total profit gained by the two leader carriers. In order to compare the individual profits the total profit gained from the complete collaboration must be split between the two carriers using the Shapley Value principle. When the joint profits are split using the Shapley Value the individual profits were not necessarily higher than partial collaboration. However, the individual profits from Shapley Value are consistently higher than the no collaboration case.

The results for the 30 node network suggest that the profits received by an individual carrier may not always increase with increasing demand. Another important factor to be noted is that the order in which the collaboration is being formed in all the cases discussed here is carrier 1 followed by the carrier 2 . With increasing demands one of the carriers may not have much to offer to the other carrier, thus resulting in reduction in the total profits for the carrier receiving less capacity from the other carrier. Thus, it is quite evident from these results that the network design, i.e., the specific arcs owned by different carriers, the network size and structure play an important role in determining the profits obtained during the collaboration process. The results for the 30 node network as explained above support this argument. 
Table 5.2 Profit Comparison: 30, 40 and 50 Node network, 2 carriers

\begin{tabular}{|c|c|c|c|c|c|c|c|c|}
\hline \multirow[t]{2}{*}{ Demand } & \multirow{2}{*}{$\begin{array}{c}\mathrm{C} 1 \\
\text { (Ind) } \\
\end{array}$} & \multirow{2}{*}{$\begin{array}{c}\text { C2 } \\
\text { (Ind) }\end{array}$} & \multicolumn{3}{|c|}{$\mathrm{C} 1+\mathrm{C} 2$ (Partial) } & \multicolumn{3}{|c|}{$\mathrm{C} 1+\mathrm{C} 2$ (Complete) } \\
\hline & & & $\mathrm{C} 1$ & $\mathrm{C} 2$ & Total & $\mathrm{C} 1$ & $\mathrm{C} 2$ & Total \\
\hline \multicolumn{9}{|c|}{30 Node Network } \\
\hline Very Low & 320 & 560 & 460 & 620 & 1080 & 420 & 660 & 1080 \\
\hline Low & 320 & 608 & 384 & 704 & 1088 & 408 & 696 & 1104 \\
\hline Medium & 320 & 640 & 320 & 760 & 1080 & 400 & 720 & 1120 \\
\hline High & 320 & 672 & 320 & 768 & 1088 & 384 & 736 & 1120 \\
\hline Very High & 320 & 720 & 320 & 780 & 1100 & 360 & 760 & 1120 \\
\hline \multicolumn{9}{|c|}{40 Node Network } \\
\hline Very Low & 536 & 552 & 624 & 784 & 1408 & 696 & 712 & 1408 \\
\hline Low & 1020 & 880 & 1180 & 980 & 2160 & 1170 & 1030 & 2200 \\
\hline Medium & 1240 & 1120 & 1240 & 1216 & 2456 & 1320 & 1200 & 2520 \\
\hline High & 1280 & 1280 & 1280 & 1480 & 2760 & 1380 & 1380 & 2760 \\
\hline Very High & 1280 & 1280 & 1280 & 1480 & 2760 & 1380 & 1380 & 2760 \\
\hline \multicolumn{9}{|c|}{50 Node Network } \\
\hline Very Low & 896 & 1200 & 1568 & 1416 & 2984 & 1418 & 1722 & 3140 \\
\hline Low & 980 & 1280 & 1712 & 1520 & 3232 & 1544 & 1844 & 3388 \\
\hline Medium & 980 & 1360 & 1800 & 1600 & 3400 & 1590 & 1970 & 3560 \\
\hline High & 980 & 1376 & 1872 & 1652 & 3524 & 1606 & 2002 & 3608 \\
\hline Very High & 980 & 1392 & 1844 & 1692 & 3536 & 1616 & 2028 & 3644 \\
\hline
\end{tabular}

One important aspect in modeling carrier collaboration (the partial scenario) is to take into consideration the order in which carriers join the collaboration. As Table 5.3 and 5.4 indicate that profits gained by a leader carrier changes with the order in which the collaboration has been formed. The difference in the profits for a single carrier goes up to $16 \%$ in certain cases. Also, the total profit obtained by the carriers in the partial collaboration keeps varying. The total profit from the partial collaboration can be either 
less than or greater than profit from the complete collaboration. Results for the 40 node network (Table 5.3) indicate that this difference varies from around $-8 \%$ to $4 \%$. This is because in complete collaboration shippers will also join together and route the commodities in the cheapest way possible for the entire system. When this occurs, even though the transportation cost decreases, the profits received by the leader carriers may also decrease. 
Table 5.3 Profit Comparison: 40 Node network, 3 carriers

\begin{tabular}{|c|c|c|c|c|c|c|c|c|}
\hline \multirow[t]{2}{*}{ Carrier } & \multicolumn{6}{|c|}{ Partial Collaboration } & \multirow{2}{*}{$\begin{array}{c}\text { No } \\
\text { Collaboration } \\
\end{array}$} & \multirow{2}{*}{$\begin{array}{c}\text { Complete } \\
\text { Collaboration } \\
\end{array}$} \\
\hline & $a-b-c$ & $a-c-b$ & $b-a-c$ & b-c-a & $c-a-b$ & $c-b-a$ & & \\
\hline \multicolumn{9}{|c|}{ Demand - Very Low } \\
\hline $\mathrm{a}$ & 1176 & 1056 & 1128 & 1180 & 1204 & 1148 & 772 & \\
\hline $\mathrm{b}$ & 1032 & 1056 & 1024 & 956 & 1036 & 1136 & 704 & \\
\hline $\mathrm{c}$ & 1168 & 1256 & 1196 & 1216 & 1120 & 1072 & 976 & \\
\hline TP* & 3376 & 3368 & 3348 & 3352 & 3360 & 3356 & 2452 & 3296 \\
\hline \multicolumn{9}{|c|}{ Demand - Low } \\
\hline $\mathrm{a}$ & 1352 & 1256 & 1240 & 1464 & 1480 & 1240 & 1020 & \\
\hline $\mathrm{b}$ & 976 & 1020 & 1020 & 936 & 1008 & 1200 & 880 & \\
\hline $\mathrm{c}$ & 1552 & 1576 & 1560 & 1544 & 1520 & 1520 & 1360 & \\
\hline TP* & 3880 & 3852 & 3820 & 3944 & 4008 & 3960 & 3260 & 3920 \\
\hline \multicolumn{9}{|c|}{ Demand - Medium } \\
\hline $\mathrm{a}$ & 1328 & 1292 & 1352 & 1448 & 1408 & 1304 & 1152 & \\
\hline $\mathrm{b}$ & 1120 & 1132 & 1144 & 1072 & 1072 & 1128 & 1024 & \\
\hline $\mathrm{c}$ & 1800 & 1848 & 1856 & 1844 & 1752 & 1832 & 1560 & \\
\hline TP* & 4248 & 4272 & 4352 & 4364 & 4232 & 4264 & 3736 & 4336 \\
\hline \multicolumn{9}{|c|}{ Demand - High } \\
\hline $\mathrm{a}$ & 1240 & 1400 & 1400 & 1464 & 1360 & 1400 & 1240 & \\
\hline $\mathrm{b}$ & 1160 & 1232 & 1152 & 1120 & 1120 & 1240 & 1120 & \\
\hline $\mathrm{c}$ & 1720 & 1728 & 1816 & 1792 & 1624 & 1680 & 1600 & \\
\hline TP* & 4120 & 4360 & 4368 & 4376 & 4104 & 4320 & 3960 & 4400 \\
\hline \multicolumn{9}{|c|}{ Demand - Very High } \\
\hline $\mathrm{a}$ & 1280 & 1280 & 1560 & 1520 & 1360 & 1600 & 1280 & \\
\hline $\mathrm{b}$ & 1560 & 1400 & 1280 & 1280 & 1400 & 1280 & 1280 & \\
\hline c & 1600 & 1720 & 1600 & 1600 & 1600 & 1520 & 1600 & \\
\hline TP & 4440 & 4400 & 4440 & 4400 & 4360 & 4400 & 4160 & 4400 \\
\hline
\end{tabular}


Table 5.4 Profit Comparison: 50 Node network, 3 carriers

\begin{tabular}{|c|c|c|c|c|c|c|c|c|}
\hline \multirow{2}{*}{ Carrier } & \multicolumn{6}{|c|}{ Partial Collaboration } & \multirow{2}{*}{$\begin{array}{c}\text { No } \\
\text { Collaboration } \\
\end{array}$} & \multirow{2}{*}{$\begin{array}{c}\text { Complete } \\
\text { Collaboration }\end{array}$} \\
\hline & $a-b-c$ & $a-c-b$ & $b-a-c$ & b-c-a & $c-a-b$ & $c-b-a$ & & \\
\hline \multicolumn{9}{|c|}{ Demand - Very Low } \\
\hline $\mathrm{a}$ & 1332 & 1332 & 1470 & 1332 & 1550 & 1332 & 594 & \\
\hline $\mathrm{b}$ & 1514 & 1298 & 1272 & 1430 & 1406 & 1468 & 930 & \\
\hline $\mathrm{c}$ & 1578 & 1896 & 1680 & 1686 & 1544 & 1600 & 1264 & \\
\hline $\mathrm{TP}$ & 4424 & 4526 & 4422 & 4448 & 4500 & 4400 & 2788 & 4338 \\
\hline \multicolumn{9}{|c|}{ Demand - Low } \\
\hline $\mathrm{a}$ & 2072 & 2016 & 2104 & 2052 & 2542 & 1948 & 896 & \\
\hline $\mathrm{b}$ & 1804 & 1676 & 1492 & 1716 & 1774 & 1936 & 1200 & \\
\hline $\mathrm{c}$ & 1788 & 2060 & 2068 & 1934 & 1700 & 1884 & 1632 & \\
\hline $\mathrm{TP}$ & 5664 & 5752 & 5664 & 5702 & 6016 & 5768 & 3728 & 5736 \\
\hline \multicolumn{9}{|c|}{ Demand - Medium } \\
\hline $\mathrm{a}$ & 2544 & 2376 & 2686 & 2484 & 3048 & 2364 & 980 & \\
\hline $\mathrm{b}$ & 1770 & 1704 & 1418 & 1790 & 1768 & 1840 & 1320 & \\
\hline $\mathrm{c}$ & 1880 & 2296 & 2416 & 2040 & 1856 & 2216 & 1856 & \\
\hline $\mathrm{TP}$ & 6194 & 6376 & 6520 & 6314 & 6672 & 6420 & 4156 & 6428 \\
\hline \multicolumn{9}{|c|}{ Demand - High } \\
\hline $\mathrm{a}$ & 2706 & 2604 & 2876 & 2648 & 3240 & 2624 & 980 & \\
\hline $\mathrm{b}$ & 1852 & 1556 & 1472 & 1792 & 1812 & 1760 & 1376 & \\
\hline $\mathrm{c}$ & 1948 & 2420 & 2444 & 2168 & 1948 & 2300 & 1920 & \\
\hline $\mathrm{TP}$ & 6506 & 6580 & 6792 & 6608 & 7000 & 6684 & 4276 & 6740 \\
\hline \multicolumn{9}{|c|}{ Demand - Very High } \\
\hline $\mathrm{a}$ & 2840 & 2660 & 2900 & 2680 & 3280 & 2750 & 980 & \\
\hline $\mathrm{b}$ & 1900 & 1400 & 1400 & 1780 & 1860 & 1720 & 1400 & \\
\hline $\mathrm{c}$ & 1960 & 2630 & 2540 & 2260 & 1960 & 2300 & 1920 & \\
\hline $\mathrm{TP}$ & 6700 & 6690 & 6840 & 6720 & 7100 & 6770 & 4300 & 6910 \\
\hline
\end{tabular}


Profits obtained from the complete collaboration case are split between the carriers based on the Shapely Value formula mentioned earlier. The results for the 40 node network are mentioned in Table 5.5. It can be seen from Table 5.6 that for the 50 node network, leader carrier ' $a$ ' always does better with partial collaboration, while leader carrier ' $c$ ' always does better with complete collaboration. Leader carrier ' $b$ ' is profited by partial collaboration at certain demand levels (low) and complete collaboration at other demand levels (medium or higher). For the 40 node network, though, lead carrier ' $a$ ' is always benefitted by complete collaboration. The best collaboration strategy for carriers ' $b$ ' and 'c' vary with the level of demand. It should be noted that the results for the partial collaboration mentioned in the tables are for a specific ordering of collaboration formation $(a-b-c)$.

Table 5.5 Profit Comparisons: Complete Collaboration vs. Partial Collaboration for a 40 node network

\begin{tabular}{|c|c|c|c|c|c|c|}
\hline Demand & \multicolumn{3}{|c|}{ Complete Collaboration } & \multicolumn{3}{c|}{ Partial Collaboration } \\
\hline & $\mathrm{a}$ & $\mathrm{b}$ & $\mathrm{c}$ & $\mathrm{a}$ & $\mathrm{b}$ & $\mathrm{c}$ \\
\hline Very Low & 1221 & 879 & 1195 & 1176 & 1032 & 1168 \\
\hline Low & 1440 & 1070 & 1410 & 1352 & 976 & 1552 \\
\hline Medium & 1568 & 1236 & 1532 & 1328 & 1120 & 1800 \\
\hline High & 1560 & 1280 & 1560 & 1240 & 1160 & 1720 \\
\hline $\begin{array}{c}\text { Very } \\
\text { High }\end{array}$ & 1473 & 1353 & 1573 & 1280 & 1560 & 1600 \\
\hline
\end{tabular}


Table 5.6 Comparison of results for Shapley Value and Partial Collaboration

\begin{tabular}{|c|c|c|c|c|c|c|}
\hline Demand & \multicolumn{3}{|c|}{40 Node } & \multicolumn{3}{c|}{50 Node } \\
\hline & $\mathrm{a}$ & $\mathrm{b}$ & $\mathrm{c}$ & $\mathrm{a}$ & $\mathrm{b}$ & $\mathrm{c}$ \\
\hline Very Low & SV & PC & SV & PC & PC & SV \\
\hline Low & SV & SV & PC & PC & PC & SV \\
\hline Medium & SV & SV & PC & PC & SV & SV \\
\hline High & SV & SV & PC & PC & SV & SV \\
\hline Very High & SV & PC & PC & PC & SV & SV \\
\hline
\end{tabular}




\section{Chapter 6}

\section{Conclusions}

Carrier collaboration is receiving increasing attention as it is an effective way of reducing transportation costs and improving profit for carriers by efficient utilization of their capacities. This paper presents two models for carrier collaboration in a shipper carrier network: partial collaboration and complete collaboration. In the partial collaboration mechanism, each leader carrier determines his individual prices on the arcs under his control. Based on the flows on his arcs, the leader carrier then offers the residual capacity to the rest of the leader carriers. The other leader carriers can then use the residual capacities to more efficiently transport their own commodities. The complete collaboration mechanism is where all leader carriers join together and determine the prices on all arcs to maximize the joint profit. The profits are then shared using the Shapley Value principle from game theory. The performance of the two collaboration mechanisms is compared against the no collaboration scenario by comparing the individual and total profit obtained for multiple networks and varying demand levels.

The results indicate that network topologies play an important role in determining the success of any collaboration strategy. The benefits from carrier collaboration were not 
found to necessarily increase with demand. Also the benefit of the partial collaboration strategy was found to depend on the order in which the coalition is formed. The complete collaboration strategy performs well but in certain cases the joint profit may even be less than the partial collaboration. This is because in complete collaboration the shippers also collaborate and route the commodities such that the total transportation cost is minimized. In such cases the savings in transportation cost need not translate to profits for the leader carrier.

\subsection{Scope for Future Research}

There is plenty of scope for expanding on the work conducted in this paper. As the number of players increases and as the complexities of the interactions between the agents increase various tools like agent based simulation must be developed. In this paper the market structure considered is a single leader carrier competing against secondary follower carriers. While this assumption maybe applicable for industries like coal, it may not be suitable for all industries. Another area which needs to be considered is developing collaboration mechanisms which are profitable when the demand and supply is uncertain.

Other constraints imposed upon the agents involved in the collaboration by market regulations need to be taken into consideration. Incorporating these constraints is a simple extension of the model explained in the research. A constant demand is assumed 
throughout the modeling process. But in reality this demand could be a function of the price. Modeling the collaboration under these circumstances is a challenging problem. 
Appendices 


\section{Appendix A}

\section{Notation}

A summary of the notations used in this paper are provided in this section for the convenience of the reader:

\section{A.1 Sets}

$G$ - Shipper - carrier network

$N-$ set of nodes

$A-$ Set of arcs

$O$ - set of all origin nodes

$D$ - set of all destination nodes

$A_{1}$ - arcs under the control of leader carrier

$A_{2}$ - arcs under the control of secondary carrier

$\Gamma^{-1}(i)$ - set of arcs entering node $i$.

$\Gamma(i)$ - set of arcs originating from node $i$.

$u_{a}$ - upstream node of arc a

$v_{a}$ - downstream node of arc a 


\title{
A.2 Carrier Specific Sets
}

\author{
$O^{m}-$ set of origin nodes for carrier $m$ \\ $D^{m}-$ set of destination nodes for carrier $m$ \\ $A_{1}^{m}$ - arcs under the control of leader carrier $m$ \\ $\mathbf{M}-$ set of leader carriers
}

\section{A.3 Parameters}

$b_{i}-$ demand/supply at node $\mathrm{i}$ in the network. A positive value indicates that the node is a supply node while a negative value implies it is a demand node.

$b^{m}$ - demand to be satisfied by shipper $m$

$c_{a}$ - unit cost of transportation on leader carrier arcs

$d_{a}-$ unit cost of transportation on secondary carrier arcs

$\operatorname{cap}_{a}$ - capacity of carrier arcs 


\section{A.4 Decision variables}

$x_{a}-$ flow on leader carrier arcs

$y_{a}-$ flow on secondary carrier arcs

$p_{a}$ - price set by the leader carrier to transport a unit flow

$\lambda$ - dual variables corresponding to flow balance constraints

$\delta$ - dual variables corresponding to capacity constraints

$P(S)$ - overall profit obtained by a coalition $\mathrm{S} \subset \mathbf{M}$

$P S_{i}$ - profit share of carrier $i$ 


\section{References}

1. R. Agarwal and O. Ergun. Network Design and Allocation Mechanisms for Carrier Alliances in Liner Shipping. Technical Report, Georgia Institute of Technology, 2007.

2. R. Agarwal and O. Ergun. Ship scheduling and network design for cargo routing in liner Shipping. Transportation Science, 42(2): 175 - 196, 2008.

3. L. Brotcorne, M. Labb'e, P. Marcotte and G. Savard. A Bi-level Model and Solution Algorithm for a Freight Tariff-Setting Problem. Transportation Science, 34(3): 289-302, 2000.

4. L. Brotcorne, M. Labbé, P. Marcotte and G. Savard. Joint design and pricing on a network. Operations Research, 56: 1104 - 1115, 2008.

5. L. Brotcorne, P. Marcotte, G. Savard, M. Wiart. Joint pricing and capacity setting problem , Advanced OR and AI Methods in Transportation, Jaszkiewicz, Kaczmarek, Zak, Kubiak (Eds), Publishing House of Poznan University of Technology, 304 - 310, 2005. 
6. J. P. Cote, P. Marcotte, and G. Savard. A bi-level modeling approach to pricing and fare optimization in the airline industry. Journal of Revenue and Pricing Management, 2(1): 23-46, 2003.

7. T.G. Crainic and G. Laporte. Planning models for freight transportation. European Journal of Operation Research, 97(3):409-438, 1997.

8. T. Esper and L. R. Williams. The value of Collaborative transportation management (CTM): Its relationship to CPFR and information technology. Transportation journal, 42(4): 55-65, 2003.

9. M. Figliozzi. Analysis and Evaluation of Incentive-Compatible Dynamic Mechanisms for Carrier Collaboration. Transportation Research Record, 1966(1): 34-40, 2006.

10. K. Fischer. Cooperative Transportation Scheduling: An Application Domain for DAI. Applied Artificial Intelligence, 10(1): 1-34, 1996.

11. C. S. Fisk and D. E. Boyce. Optimal Transportation Systems Planning with integrated Supply and Demand Models. Transportation Panning Group, Department of Civil Engineering, University of Illinois at Urbana-Champaign, $16,1983$. 
12. M. Florian and M. Los. A new look at static spatial price equilibrium models. Regional Science and Urban Economics, 12(4):579-597, 1982.

13. T. Friesz, R. Tobin, T. Smith, and P. Harker. A nonlinear complementarity formulation and solution procedure for the general derived demand network equilbrium problem. Journal of Regional Science, 23(3): 337-359, 1983.

14. T. Friesz, J. Gottfried, and E. Morlok. A sequential shipper-carrier network model for predicting freight flows. Transportation Science, 20(2): 80-91, 1986.

15. D. Goodwill. Shipper - Carrier Collaboration, a Myth or Reality? (2007) http://blogdg.ctl.ca/2007/04/shipper_carrier_collaboration_1.html (accessed 03/23/2009).

16. P. Guglar and J. Dunning. Technology Based Cross-Border Alliances. Multinational Strategic Alliances, R. Culpin (ed.), Binghampton, NY, Howarth Press, Inc., 1993.

17. P.T. Harker and T. L. Friesz. A simultaneous freight network equilibrium model. Cong. Numer, 36: 365 - 402, 1982. 
18. P.T. Harker and T. L. Friesz. The use of Equilibrium Network Models in Logistics Management: With Application to the US Coal industry. Transportation Research Part B, 19: 457-470, 1985.

19. P.T. Harker and T. L. Friesz. Prediction of Intercity Flows, I: Theory. Transportation Research Part B, 20: 80-91, 1986.

20. W. H. Hurley. A Theory of Rail Freight Pricing. Unpublished Ph. D. dissertation School of Business, Queen's University, Kingston, Ontario, 1988.

21. W. Hurley and E. R. Petersen. Nonlinear Tariffs and Freight Network Equilibrium. Transp. Sci., 28: 236-245, 1994.

22. A. Jean-Franois, S. D. Amours, and M. Ranqvist. Business Models for Collaborative Planning in Transportation: an Application to Wood Products. http://www.ebrc.fi/kuvat/Audy DAmours Ronnqvist paper.pdf (accessed 10/01/2009).

23. D. Klingman, A. Napier, and J. Stutz. NETGEN: A Program for Generating Large Scale Capacitated Assignment, Transportation, and Minimum Cost Flow Network Problems. Management Science, 20(5): 814-821, 1974. 
24. M. A. Krajewska and H. Kopfer. Collaborating freight forwarding enterprises. $O R$ Spectrum, 28(3): 301-317, 2006.

25. V. Krishna. Auction Theory. Academic Press, 2002.

26. L. Lei, C. Fan, M. Boile, and S. Theofanis. Collaborative vs. non-collaborative container vessel Scheduling. Transportation Research Part E, 44(3): 504-520, 2008.

27. P. Marcotte. Algorithms for the network oligopoly problem. Journal of the Operational Research Society, 38(11): 1051-1065, 1987.

28. P. Marcotte, G. Savard, and D. Zhu. Mathematical structure of a bi-level strategic pricing Model. European Journal of Operational Research, 193(2): 552 - 566, 2009.

29. R. B. Myerson and M. A. Satterthwaite. Efficient mechanisms for bilateral trading. Journal of Economic Theory, 29(2): 265-281, 1983.

30. T. Nemoto. Area-wide inter-carrier consolidation of freight in urban areas. Transport Logistics, 1(2): 87-101, 1997. 
31. M. Owoc and M. A. Sargious. The role of transportation in free trade competition. Canadian Transportation: Competing in a Global Context, 23-32, 1992.

32. E. Plant and J. McGovern (2007). Rationales for collaboration; implications for the Irish road freight industry.Dublin Institute of Technology. http://arrow.dit.ie/engschmeccon/8/ (accessed 03/23/2009).

33. P.Rey and J. Tirole. The Logic of Vertical Restraint. American Economic Review, 76: 921-39 1986.

34. L. Shapley. A value for n-person games. Classics in Game Theory, 1997.

35. J. Song and A. Regan. An Auction Based Collaborative Carrier Network. Transportation Research, Part E, under review, 2003.

36. E. Strocko. Making the Case for Freight Investments. Public Roads, 71(6), 2008. http://www.tfhrc.gov/pubrds/08may/03.htm (accessed 02/10/2009).

37. J.Tirole. A Theory of Industrial Organization, MIT Press, 1989.

38. USDOT and FHWA. Creating a Freight sector within HERS. White Paper, 2001. 


\section{Vita}

Avinash Voruganti, son of Gnaneshwar Voruganti and Swayam Prabha Voruganti, was born in Hyderabad, India, on 27 November, 1985. He began his undergraduate studies at the Indian Institute of Technology Madras, in August 2003. He received the Bachelor of Science degree in Civil Engineering from the Indian Institute of Technology Madras, in July 2007. He began pursuing his graduate studies in Transportation Engineering at the University of Texas at Austin in the fall of 2007.

Permanent address: Ho. No. 7-145/1, Nagendranagar Colony, Habsiguda, Hyderabad, India 500007 